\title{
Synchrotron-Based Data-Constrained Modeling Analysis of Microscopic Mineral Distributions in Limestone
}

\author{
Yudan Wang ${ }^{1}$, Yushuang Yang ${ }^{2 *}$, Tiqiao Xiao ${ }^{1 *}$, Keyu Liu ${ }^{3,4}$, Ben Clennell ${ }^{3}$, \\ Guoqiang Zhang ${ }^{1}$, Haipeng Wang \\ ${ }^{1}$ Shanghai Institute of Applied Physics, Chinese Academy of Sciences, Shanghai, China \\ ${ }^{2}$ Materials Science \& Engineering, Commonwealth Scientific and Industrial Research Organization, Clayton, Australia \\ ${ }^{3}$ Earth Science \& Resource Engineering, Commonwealth Scientific and Industrial Research Organization, Bentley, Australia \\ ${ }^{4}$ Research Institute of Petroleum Exploration and Development, PetroChina, Beijing, China \\ ${ }^{5}$ College of Physics \& Electronics Engineering, Shanxi University, Taiyuan, China \\ Email: ${ }^{*}$ sam.yang@csiro.au, ${ }^{*}$ tqxiao@sinap.ac.cn
}

Received November 9, 2012; revised December 16, 2012; accepted January 17, 2013

\begin{abstract}
Three dimensional (3D) microscopic distributions of dolomite and calcite in a limestone sample have been analyzed with a data-constrained modeling (DCM) technique using synchrotron radiation-based multi-energy X-ray computed tomography $(\mathrm{CT})$ data as constraints. In order to optimize the experimental parameters, X-ray CT simulations and DCM analysis of a numerical phantom consisting of calcite $\left(\mathrm{CaCO}_{3}\right)$ and dolomite $\left(\mathrm{CaMg}\left(\mathrm{CO}_{3}\right)_{2}\right)$ have been used to investigate the effects on the predicted results in relation to noise, X-ray energy and sample-to-detector distance (SDD). The simulation results indicate that the optimal X-ray energies are 25 and $35 \mathrm{keVs}$, and the SDD is $10 \mathrm{~mm}$. The high resolution 3D distributions of mineral phases of a natural limestone have been obtained. The results are useful for quantitative understanding of mineral, porosity, and physical property distributions in relation to oil and gas reservoirs hosted in carbonate rocks, which account for more than half of the world's conventional hydrocarbon resources. The case studied is also instructive for the applicability of the DCM methods for other types of composite materials with modest atomic number contrasts between the mineral phases.
\end{abstract}

Keywords: X-Ray Computed Tomography (CT); Synchrotron Radiation (SR); Data-Constrained Modeling (DCM); Microstructure; Limestone

\section{Introduction}

Knowledge of three dimensional (3D) material distributions for hydrocarbon reservoir limestone at microscopic length scales is important in understanding their mechanical and hydrological properties in relation to oil and gas production and carbon dioxide $\left(\mathrm{CO}_{2}\right)$ geo-sequestration. Traditional methods such as optical and scanning electron microscopy are common tools for providing valuable information of microstructures, for quantitative modeling investigations on microstructure/property relationship of various materials [1-3]. However, those surface observations are often inadequate in obtaining detailed 3D information of the sample, such as compositional distribution inside limestone. A sample-destructive direct 3D analytical method is serial sectioning [4-6]. Nevertheless, the materials between sections are destroyed with only the serial sectioned images remaining.

$\mathrm{X}$-ray computed tomography $(\mathrm{CT})$ is a non-destructive method that provides sectional X-ray attenuation images

"Corresponding author.
(CT images) of objects. The synchrotron radiation (SR) source provides energy-tunable, monochromatized, and naturally collimated X-ray beams that have many advantages for CT. Monochromatized beams eliminate beam hardening, which causes $\mathrm{CT}$ image artifacts, and thus permit $\mathrm{CT}$ values to relate quantitatively to X-ray linear attenuation coefficients. Furthermore, the synchrotronbased X-ray CT has the advantage of high spatial resolution and has been widely used in characterization of internal structures of a diverse range of materials in biological, medical, materials science and geoscience [7-11]. However, as different compositions of materials may exhibit similar X-ray absorption and refraction characteristics, it is often inadequate to resolve material compositions. A data-constrained modelling (DCM) methodology has been developed for characterising compositional microstructures using two or more CT datasets acquired with different X-ray spectra and incorporates them as model constraints [12-14]. For instance, the effect of nano-porous regions or partially occupied voxels can be identified. The advantage of the DCM approach 
includes its capability in resolving partial occupancy of multiple material compositions at the voxel level samplenon-destructively. Such partial occupancy accounts for the effects below normal CT resolution. The DCM approach has been successfully applied to characterize and predict the microstructure of a range of different materials including: the distribution of corrosion inhibitor particles in aerospace paint primers $[15,16]$, zinc corrosion product distributions [17], gold particle distributions in nano-structured functional materials [18], and mineral phases distributions in hydrocarbon reservoir sandstones [19,20].

In this article, we have applied high-resolution multispectrum X-ray CT technique combined with a DCM approach to study a natural carbonate (limestone) sample containing predominantly calcite $\left(\mathrm{CaCO}_{3}\right)$ and dolomite $\left(\mathrm{CaMg}\left(\mathrm{CO}_{3}\right)_{2}\right)$ mineral phases. The quantitative effects of experimental parameters on prediction accuracy and noise immunity have been studied using simulated numerical phantoms. The optimal experimental parameters and credibility of prediction for each material in the sample have been analyzed. X-ray CT experiments were performed at X-ray Imaging and Biomedical Application (BL13W) beamline at the Shanghai Synchrotron Radiation Facility (SSRF). The 3D distribution maps of mineral phases in the sample with high resolution were obtained with DCM.

\section{Simulation Analysis of Numerical Phantoms}

This section is focused on evaluation of the impacts of experimental variables on X-ray CT attenuation map and DCM composition map reconstruction accuracies. The evaluation is conducted with a numerical phantom with $512 \times 512 \times 32$ voxels (a voxel has a size of $3.7 \times 3.7 \times$ $3.7 \mu \mathrm{m}^{3}$ ), as shown in Figure 1. The numerical phantom was divided into sub-regions which were comprised of different proportions of calcite and dolomite with the volume fraction step of $10 \%$. From the left to right and the bottom to up, the volume fractions of calcite are $100 \%, 90 \%, 80 \%, 70 \%, 60 \%, 50 \%, 50 \%, 40 \%, 30 \%$, $20 \%, 10 \%$ and $0 \%$. The remainders of the cylinders are dolomite. The minerals in the phantom include calcite and dolomite, as listed in Table $\mathbf{1 .}$

Projection images of the phantom have been simulated with monochromatic parallel beams at X-ray energies of $25 \mathrm{keV}, 30 \mathrm{keV}, 35 \mathrm{keV}$ and $38 \mathrm{keV}$. The sample-to- detector distances (SDDs) were set to $10 \mathrm{~mm}, 100 \mathrm{~mm}$ and $200 \mathrm{~mm}$. At each discrete combination of X-ray energy and SDD, 360 projections over a total rotation angle of $180^{\circ}$ were obtained with a $0.5^{\circ}$ angular step between projections. The projection images correspond mathematically to the Radom transforms of the phantom with $\mathrm{X}$-ray absorption and refraction. The images contain phase-contrast effects for non-zero SDD. That is, the projection images recorded by the detector were intensity images of the sample after Fresnel propagation at a distance of SDD. For purpose of comparison, a zero SDD case was simulated, although it cannot be achieved in practice.

In order to investigate the effect of noise, various levels of Gaussian noise were added to the simulated X-ray projections at levels of $0.1 \%, 0.2 \%, 0.5 \%, 1 \%, 1.5 \%$ and $2 \%$. The actual experimental noise level was also used to simulate the projection images. The measured experimental standard deviation values were $1.21 \%$ at $25 \mathrm{keV}$, $1.15 \%$ at $30 \mathrm{keV}, 1.17 \%$ at $35 \mathrm{keV}$ and $1.72 \%$ at $38 \mathrm{keV}$.

After the simulated projections were obtained, images were subjected to phase-retrieval processing prior to tomographic reconstruction. Phase-retrieval processing was a result of the phase-contrast imaging mode which exhibited an apparent improvement in signal-to-noise level. The resulting tomographic cross sections resemble those from a conventional micro-CT. Slices of X-ray

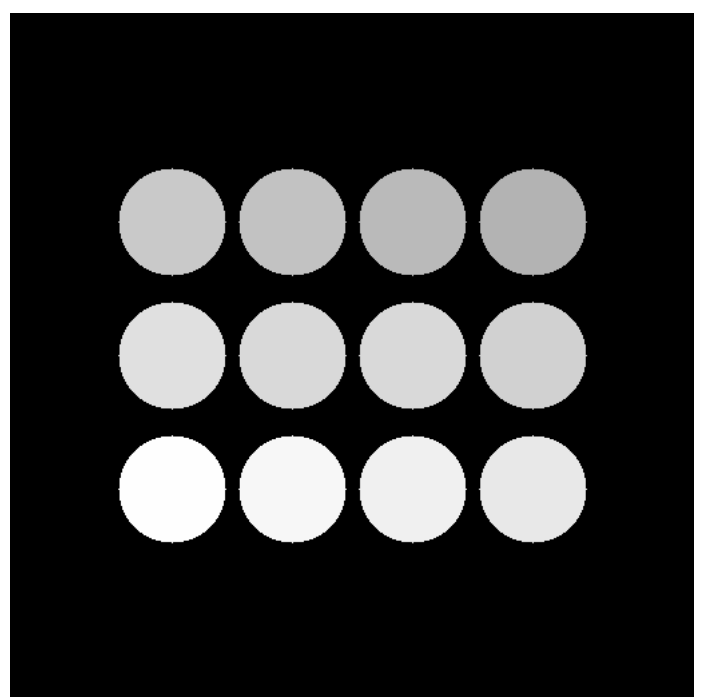

Figure 1. The simulated numerical phantom with various proportions of calcite and dolomite (the volume fraction

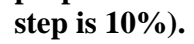

Table 1. The total linear absorption indices $(\beta)$ of calcite and dolomite at the energies of $25 \mathrm{keV}, 30 \mathrm{keV}, 35 \mathrm{keV}$ and $38 \mathrm{keV}$.

\begin{tabular}{|c|c|c|c|c|c|c|}
\hline \multirow{2}{*}{ Material } & \multirow{2}{*}{ Formula } & \multirow{2}{*}{ Density $\left(\mathrm{g} / \mathrm{cm}^{3}\right)$} & \multicolumn{4}{|c|}{ Total linear absorption indices $(\beta)$} \\
\hline & & & $25 \mathrm{keV}$ & $30 \mathrm{keV}$ & $35 \mathrm{keV}$ & $38 \mathrm{keV}$ \\
\hline Calcite & $\mathrm{CaCO}_{3}$ & 2.71 & $3.16 \times 10^{-9}$ & $1.60 \times 10^{-9}$ & $9.18 \times 10^{-10}$ & $6.88 \times 10^{-10}$ \\
\hline Dolomite & $\mathrm{CaMg}\left(\mathrm{CO}_{3}\right)_{2}$ & 2.87 & $2.21 \times 10^{-9}$ & $1.14 \times 10^{-9}$ & $6.73 \times 10^{-10}$ & $5.11 \times 10^{-10}$ \\
\hline
\end{tabular}


attenuation information at a given X-ray energy were obtained by using the filtered back-projection algorithm as the $\mathrm{CT}$ reconstruction method.

The DCM linear optimization approach was then used to reconstruct the compositional maps. On each voxel at position $i$, the DCM approach $[19,20]$ can be expressed as

$$
\left\{\begin{array}{l}
0 \leq v_{i}^{(p o r)}, v_{i}^{(c a l)}, v_{i}^{(d o l)} \leq 1 \\
v_{i}^{(p o r)}+v_{i}^{(c a l)}+v_{i}^{(d o l)}=1 \\
\beta_{i, E}^{(p o r)} v_{i}^{(p o r)}+\beta_{i, E}^{(c a l)} v_{i}^{(c a l)}+\beta_{i, E}^{(d o l)} v_{i}^{(d o l)}=\bar{\beta}_{i, E}, E=E_{1}, E_{2}, \cdots
\end{array}\right.
$$

where $v_{i}^{(p o r)}, v_{i}^{(c a l)}$ and $v_{i}^{(d o l)}$ denote for the volume fractions for pore, calcite and dolomite respectively; $\bar{\beta}_{i, E}$ is the absorption indices in reconstructed slices at the X-ray beam energy of $E$; and $\beta_{i, E}^{(p o r)}, \beta_{i, E}^{(c a l)}$ and $\beta_{i, E}^{(d o l)}$ are the absorption indices for pore, calcite and dolomite at the X-ray beam energy of $E$ respectively [20]. Equation (1) constitutes a linear optimization problem with non-negativity constraints. It is solved using the DCM software with the Simplex algorithm [21]. The solutions of the values $v_{i}^{(p o r)}, v_{i}^{(c a l)}$ and $v_{i}^{(d o l)}$ for all voxels form a predicted compositional map of the original phantom. For each discrete combination of SDD and noise level, a DCM predicted 3D set of images was obtained.

In order to evaluate the performance of multi-energy $\mathrm{X}$-ray CT and DCM under different experimental conditions, the normalized cross-correlation (NCC) between the predicted image and the original volume fractions for each material was calculated

$$
C=\frac{\sum_{i=1}^{N}\left(v_{i}^{(p)}-\bar{v}^{(p)}\right)\left(v_{i}^{(o)}-\bar{v}^{(o)}\right)}{\left[\sum_{i=1}^{N}\left(v_{i}^{(p)}-\bar{v}^{(p)}\right)^{2} \sum_{i=1}^{N}\left(v_{i}^{(o)}-\bar{v}^{(o)}\right)^{2}\right]^{1 / 2}}
$$

where $v_{i}^{(p)}$ and $v_{i}^{(o)}$ are DCM-predicted and original volume fraction values of the material at $i$ th voxel respectively, while $\bar{v}^{(p)}$ and $\bar{v}^{(o)}$ are their mean values. The NCC represents a template matching of two datasets, which means that a higher NCC value corresponds to a closer match between the predicted image and the real sample.

Table 2 shows the NCC values at different energy combinations and a SDD of $10 \mathrm{~mm}$ at experimental noise level and the condition numbers [21] of the coefficient matrix in Equation (1). From Table 2, it is shown that the best X-ray energy combination for the limestone sample is at $25 \mathrm{keV}$ and $35 \mathrm{keV}$. This is different from the energy combination at $25 \mathrm{keV}$ and $38 \mathrm{keV}$ which gives the smallest condition number. The discrepancy is due to the fact that in this paper, it is assumed that the noise level is the same for projection images at different X-ray beam
Table 2. The NCCs at different energy combinations at SDD of $10 \mathrm{~mm}$ and the condition numbers of the coefficient matrix in Equation (1).

\begin{tabular}{cccc}
\hline & \multicolumn{2}{c}{$\begin{array}{c}\text { Normalized } \\
\text { Energy/keV }\end{array}$} & \multicolumn{2}{c}{ Cross-Correction (NCC) } & $\begin{array}{c}\text { Condition } \\
\text { Number }\end{array}$ \\
\cline { 2 - 3 } & $\mathrm{CaCO}_{3}$ & $\mathrm{CaMg}\left(\mathrm{CO}_{3}\right)_{2}$ & \\
\hline $25 \& 30$ & 0.88 & 0.70 & 256 \\
$25 \& 35$ & 0.89 & 0.72 & 142 \\
$25 \& 38$ & 0.89 & 0.70 & 120 \\
$30 \& 35$ & 0.86 & 0.65 & 195 \\
$30 \& 38$ & 0.87 & 0.66 & 127 \\
$35 \& 38$ & 0.85 & 0.63 & 274 \\
$25 \& 30 \& 35$ & 0.88 & 0.59 & N/A \\
$25 \& 30 \& 38$ & 0.88 & 0.56 & N/A \\
30 \& $35 \& 38$ & 0.86 & 0.51 & N/A \\
\hline $25 \& 30 \& 35 \& 38$ & 0.87 & 0.62 & N/A \\
\hline
\end{tabular}

energies. The estimation with the matrix condition number has an implicit assumption that the noise level is the same with the CT reconstructed absorption indices [18].

The above analysis uses 2 sets of CT data. In order to know whether the accuracy would be improved with additional sets of $\mathrm{CT}$ data at other X-ray energies, we also included 3 and 4 sets of CT data as shown in Table 2. Instead of improvement, inclusion of additional data set has shown a decrease in prediction accuracy. This could be related to the fact that the data sets have a low degree of linear independence as suggested by the relatively large condition number. Separate datasets may have different zero offset due to experimental noise. Further investigations are required to fully understand the phenomena.

Figure 2 shows that the NCC values decrease with noise level for fixed values of X-ray beam energy and SDDs. This is expected as the DCM reconstruction accuracy decreases with increasing noise. The figure also indicates that the reconstruction accuracy is very sensitive to noise for small SDD. This is particularly true at $\mathrm{SDD}=0 \mathrm{~mm}$. The noise immunity increases at higher values of SDD. Figure 3 shows the relation between SDD and NCC at beam energies of $25 \mathrm{keV} \& 35 \mathrm{keV}$ and at the experimental noise level. In the figure, the solid line is for calcite and the dashed line is for dolomite. It has been observed that the NCC value first increases and then decreases with SDD. From Figures 2 and 3, NCC appears to be small and sensitive to noise at SDD = $0 \mathrm{~mm}$. This is because the slices were reconstructed directly from projections. As SDD increases from zero, NCC increases. This is owing to the fact that phase 


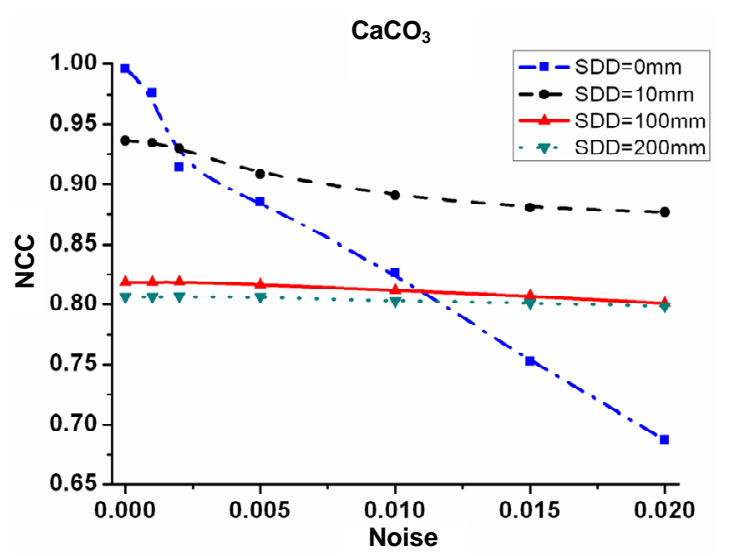

(a)

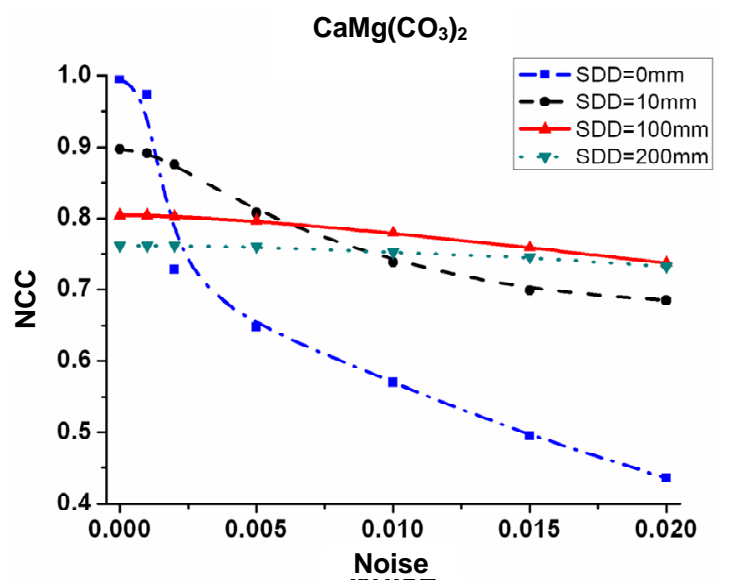

(b)

Figure 2. The graph of the NCC at $25 \mathrm{keV} \& 35 \mathrm{keV}$ at a series of SDDs as a function of the noise.

retrieval processing was applied to the non-zero SDD case which could improve the signal-to-noise of the CT reconstructions. When SDD takes higher values, NCC reaches a maximum then decreases with increasing SDD. This is because a larger SDD induces a higher level of Fresnel diffraction and edge enhancement effect, which will affect the reconstructed absorption indices [22]. The noise level is usually in the range of $1 \%$ to $2 \%$ in the experiment. From Figure 2, the NCC at SDD of $10 \mathrm{~mm}$ reaches its highest value for calcite, while for dolomite the maximum NCC is obtained at a SDD of $100 \mathrm{~mm}$. Figure 3 also shows that the best SDDs for calcite and dolomite are found to be $10 \mathrm{~mm}$ and $100 \mathrm{~mm}$ respectively, consistent with the analysis above. For a sample composed of both calcite and dolomite, the optimal SDD is the one that gives the highest value of NCC. Figures 2 and 3 indicate that the optimal SDD is $10 \mathrm{~mm}$.

\section{DCM Characterization of a Limestone Sample}

The natural limestone sample used for experiments

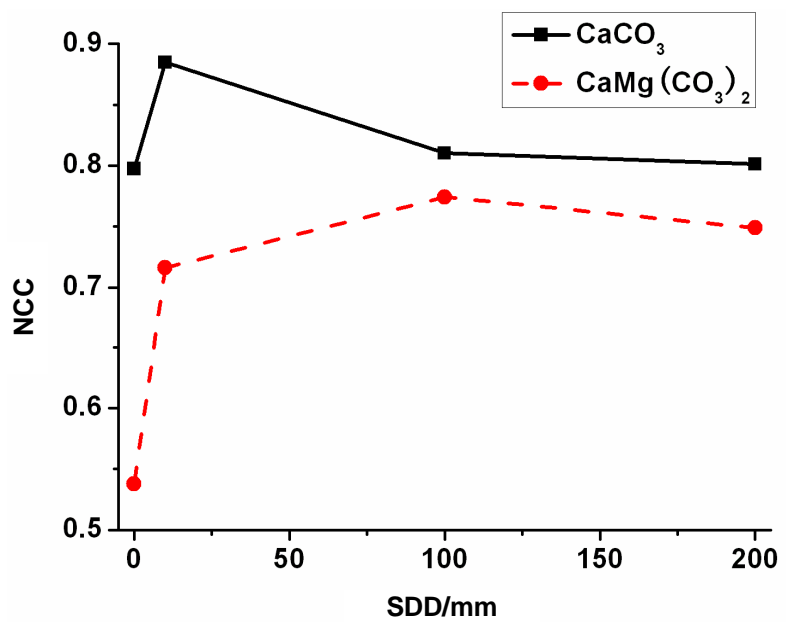

Figure 3. The relation of SDD and NCC at $25 \mathrm{keV} \& 35 \mathrm{keV}$ at experimental noise.

consists of calcite and dolomite. In this rock, the two minerals were distinct, and can be well differentiated by electron microscopy incorporating back-scattered electron contrast. This provided a good basis for quantifying the success of the X-ray based methods. The limestone sample was from the Triassic Feixianguan (T1f) Formation from the Zhujia-1 well $(5578.7 \mathrm{~m})$ in the northeastern Sichuan Basin, South China. The Feixianguan Formation was a major reservoir interval in the basin and hosts a number of gas fields including the giant Puguang Gas Field. T1f was deposited as inter-bedded oolitic limestone and calcareous marl in a restricted marine (lagoonal) environment associated with gypsum deposition on a carbonate platform margin [23]. Multiple dolomitisation occurred during the post depositional diagenesis [24]. For high resolution imaging, a cylindrical plug was prepared with a diameter of about $3.5 \mathrm{~mm}$.

The X-ray micro-tomography experiments were carried out at BL13W beamline at SSRF in Shanghai, China. Micro-tomography is composed of an X-ray source, a sample stage, a double-crystal monochromator and a charge-coupled device (CCD) system, as shown in Figure 4 . $\mathrm{BL} 13 \mathrm{~W}$ is a wiggler beamline producing monochromatic X-rays with a flux density of $\sim 3.4 \times 10^{10}$ photons $/ \mathrm{s} / \mathrm{mm}^{2} @ 20 \mathrm{keV}$ and narrow energy band pass $(\Delta \mathrm{E} / \mathrm{E}$ $<5 \times 10^{-3}$, where $E$ is the photon energy) by an $\mathrm{Si}(111)$ double-crystal monochromator. As suggested by the simulation results in the previous section, $\mathrm{X}$-ray energies were set to $25 \mathrm{keV}$ and $35 \mathrm{keV}$ and SDD was chosen as $10 \mathrm{~mm}$. An Optique Peter X-ray CCD detector was used to acquire images, which has a native pixel size of $7.4 \times$ $7.4 \mu \mathrm{m}^{2}$. Combined with a $2 \times$ optical lens, it has provided an effective isotropic voxel with a $3.7 \mu \mathrm{m}$ edge. For each X-ray beam energy, 1080 projection images were acquired with a $0.167^{\circ}$ angular spacing between each view. For background correction, flat-field images 


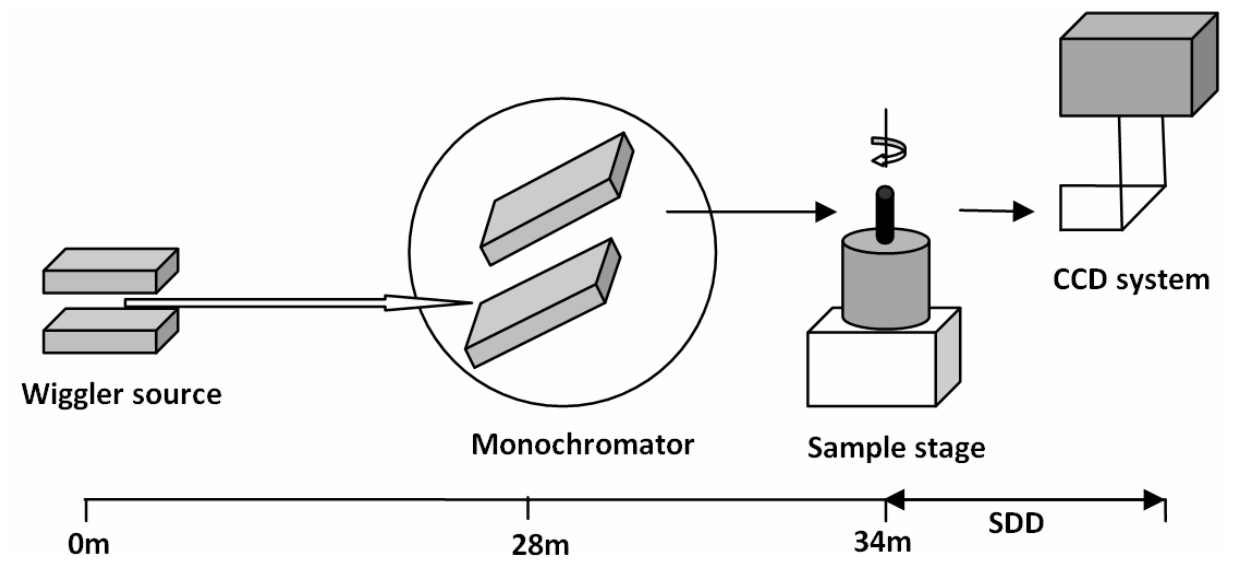

Figure 4. A schematic of the micro CT system at BL13W of SSRF used in the present experiments.

of direct X-ray beams were measured with no sample for every ten degrees during the sample scanning to correct for time decay of the X-ray intensity. Dark current of the detector system was measured after the CT measurement for correction of the images. The exposure time was 1.5 seconds at both $25 \mathrm{keV}$ and $35 \mathrm{keV}$ beam energies.

The CT slices were reconstructed using the X-TRACT software package [25]. Background corrections were applied using flat-field and dark-current images. Figure 5 is a typical projection image after background correction at $35 \mathrm{keV}$ at SDD of $10 \mathrm{~mm}$. Image normalization was performed to compensate the uneven beam within the image field. Next, the normalized images were subject to Paganin's phase-retrieval processing prior to tomographic reconstruction. Paganin's phase-retrieval algorithm was applied with parameters tuned to optimize the signal-to-noise and minimize the edge enhancement without loss of resolution [26]. Finally, the slices were reconstructed by using the method of filtered back-projection of parallel X-ray beam. Since there were some ring artifacts in the slices, a ring artifacts correction based on polar coordinate transformation was performed for these slices. After that, image alignment of data sets for the two slices at different energies was achieved by translation and rotation of slices to maximise the crosscorrelations between corresponding images. The processed slices so produced, containing information of absorption indices were used for further analysis by using the DCM method.

A typical slice (Slice 500) at $35 \mathrm{keV}$ and its histogram are shown in Figures 6(a) and (b), respectively. There are three peaks in the histogram which correspond to airfilled pores, dolomite and calcite with the $\beta$ values of $7.99 \times 10^{-12} \pm 1.1 \times 10^{-10}, 6.35 \times 10^{-10} \pm 1.45 \times 10^{-10}$ and $8.31 \times 10^{-10} \pm 3 \times 10^{-11}$, respectively. Similarly, the $\beta$ values of the slice at $25 \mathrm{keV}$ for pores, dolomite and calcite were obtained. Their values were $2.84 \times 10^{-11} \pm 2.42$ $\times 10^{-10}, 1.99 \times 10^{-9} \pm 3.96 \times 10^{-10}$ and $2.58 \times 10^{-9} \pm 9 \times$

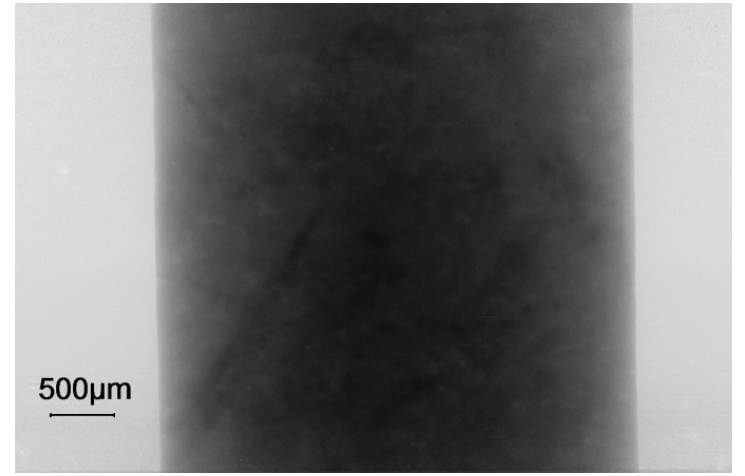

Figure 5. A typical projection image after background correction at $35 \mathrm{keV}$ at SDD of $10 \mathrm{~mm}$.

$10^{-11}$, respectively. The reconstructed attenuation values were lower than that expected for the two material phases. The discrepancy may be caused by a number of factors including the point-spread function (PSF) of the imaging system, which is induced by source size, the PSF of detector, the geometry of the system and so on [27]. The CT reconstructed absorption coefficients were corrected by linear rescaling of the reconstructed slices and shifting of pores values. The CT datasets for both beam energies of $25 \mathrm{keV}$ and $35 \mathrm{keV}$ have been rescaled by a multiplication factor of 1.1, whilst the shifting of pores values for the $25 \mathrm{keV}$ and $35 \mathrm{keV}$ beam energies are $8 \times 10^{-11}$ and $-6 \times 10^{-11}$, respectively.

Using the above two CT datasets as constraints, a DCM linear optimization approach was used to obtain compositional distributions of calcite and dolomite [19]. The mineral phases of the slice (Slice 500) with the size of $1119 \times 1080$ pixels were obtained. The distributions of dolomite and calcite have been extracted from the sample, as shown in Figures 7(a) and (b), respectively. The pixel intensities in these figures denote the volume fractions of the corresponding mineral phases. The DCM calculated 3D compositional distributions of dolomite and calcite on a cubic grid of $500 \times 500 \times 300$ pixels inside the sample 


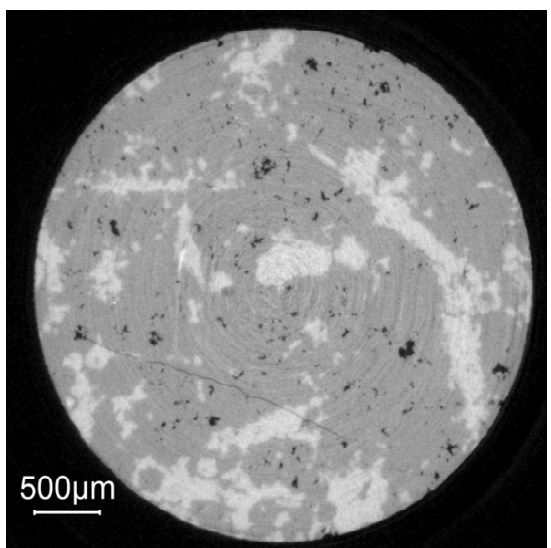

(a)

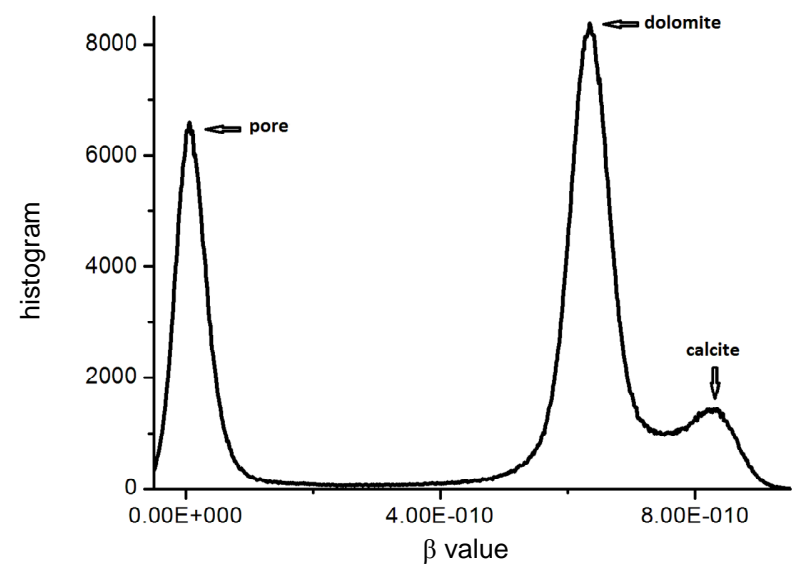

(b)

Figure 6. A typical X-ray CT reconstructed slice (slice 500) at $35 \mathrm{keV}$ at SDD of $10 \mathrm{~mm}$.

are shown in Figures 8(a) and (b), respectively.

It is shown in Figures $\mathbf{7}$ and $\mathbf{8}$ that a fraction of calcite formed clusters inside dolomite, and pores was concentrated in some parts of the sample. The figures also indicate that there was a significant proportion of pores and dolomite which were smaller than the CT resolution (3.7 $\mu \mathrm{m})$, which is shown as unsaturated pixel intensity for images. The volume fractions of calcite, dolomite and pores were calculated as average voxel values. In the part of the sample shown in Figure 8, their values are 15\%, $81 \%$ and $4 \%$, respectively. The correct value of porosity and the correct assignment of the pores within the 3D volume is very important for the computation of rock properties such as fluid permeability and elastic constants, which are important for exploitation of carbonate oil and gas reservoirs.

\section{Conclusions}

Microscopic 3D distributions of calcite and dolomite in a limestone sample were studied with a data-constrained modeling (DCM) approach using multi-spectrum quanti-

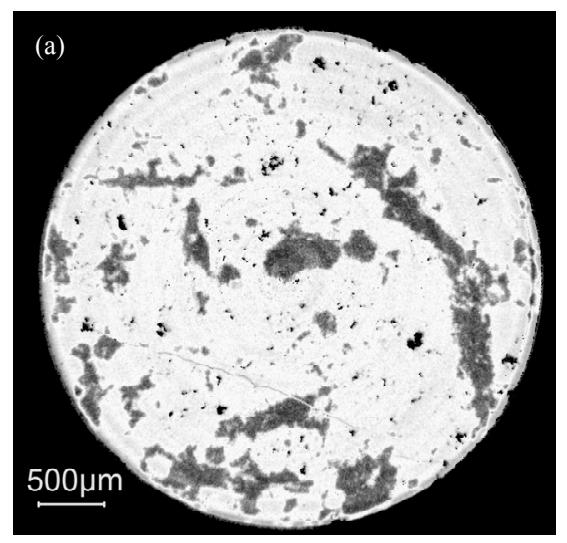

(a)

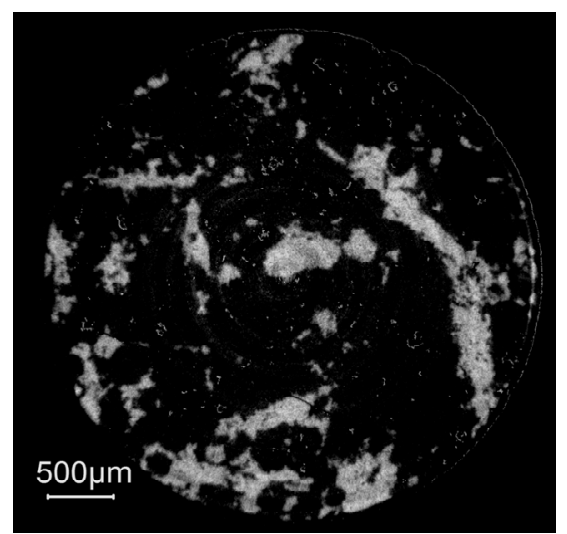

(b)

Figure 7. Distribution of mineral phases on the same $X-Y$ plane (slice 500) as in Figure 6, (a) is dolomite and (b) is calcite.

tative X-ray CT data as model constraints. A simulated numerical phantom with various ratios of calcite and dolomite was used to investigate noise sensitivity, effects of X-ray beam energy and sample-to-detector distance (SDD). The simulation results show that the prediction accuracy decreases with increasing noise, the optimal compromise for SDD is $10 \mathrm{~mm}$ and the optimal X-ray energies are $25 \mathrm{keV}$ and $35 \mathrm{keV}$ which were selected as the experimental distance and energies. The optimized parameters are useful for future analysis of the microstructures of similar samples.

A natural limestone sample was prepared and synchrotron-based monochromatic multi-energy X-ray CT experiments have been carried out at SSRF with parameters of X-ray beam energies at $25 \mathrm{keV}$ and $35 \mathrm{keV}$ and SDD at $10 \mathrm{~mm}$ as simulated. DCM characterisation of the microscopic distribution of mineral phases was obtained from multi-energy quantitative X-ray CT reconstructed image sets. The results indicated that the pore was concentrated in some parts of the sample, and a proportion of pores and dolomite had sizes smaller than the $\mathrm{CT}$ resolution. It has been demonstrated that by using 


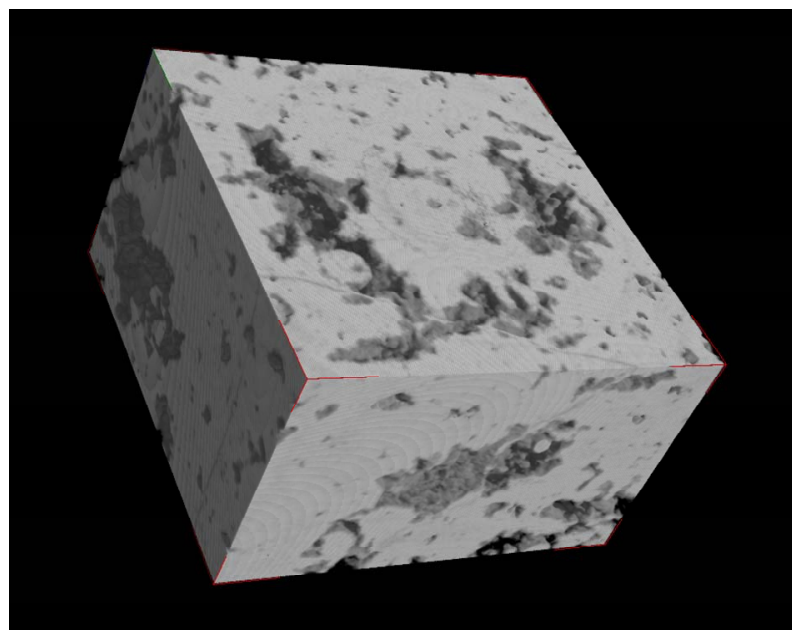

(a)

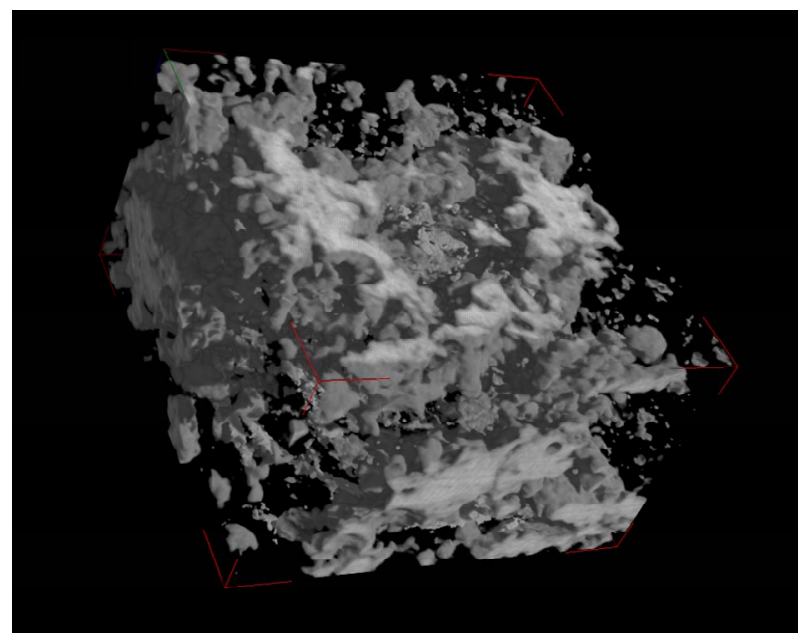

(b)

Figure 8. The three dimensional compositional distribution of part of sample, (a) is dolomite and (b) is calcite.

multiple energies X-ray CT and the DCM method, a segmentation for mineral phases and pore space could be achieved. The DCM method enables us to visualize the distribution not only of discrete, resolved pores, but also regions of calcite and dolomite minerals that are micro-porous. The investigation has demonstrated that the method is a powerful tool for quantitative analysis of geological samples with complex mineralogy where the single energy X-ray tomography method fails to produce an adequately segmented rock volume.

\section{Acknowledgements}

Y. D. Wang would like to acknowledge sponsorship by the Chinese Scholarship Council (CSC) and hospitality by CSIRO Material Science \& Engineering Division during which this work has been carried out. Y. S. Yang would like to acknowledge support by the One Hundred Person Project of Shanxi Province. The authors would also like to acknowledge support by the CSIRO Advanced Materials and Computational and Simulation Science Transformational Capability Platforms (AM-TCP and CSS-TCP).

\section{REFERENCES}

[1] H. Andersson and C. Persson, "In-Situ SEM Study of Fatigue Crack Growth Behaviour in IN718," International Journal of Fatigue, Vol. 26, No. 3, 2004, pp. 211219. doi:10.1016/S0142-1123(03)00172-5

[2] C. Motz and R. Pippan, "Fracture Behaviour and Fracture Toughness of Ductile Closed-Cell Metallic Foams," Acta Materialia, Vol. 50, No. 8, 2002, pp. 2013-2033. doi:10.1016/S1359-6454(02)00047-2

[3] L. H. Qian, H. Toda, S. Morita, T. Kobayashi and Z. G. Wang, "In-Situ Observations of Fracture Processes in 0.6

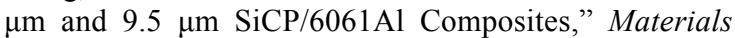
Transactions, Vol. 46, No. 1, 2005, pp. 34-41. doi:10.2320/matertrans.46.34

[4] D. N. Bryon, M. P. Atherton and R. H. Hunter, "The Interpretation of Granitic Textures from Serial Thin Sectioning, Image-Analysis and 3-Dimensional Reconstruction," Mineralogical Magazine, Vol. 59, No. 395, 1995, pp. 203-211. doi:10.1180/minmag.1995.059.395.05

[5] R. Marschallinger, "Correction of Geometric Errors Associated with the 3-D Reconstruction of Geological Materials by Precision Serial Lapping," Mineralogical Magazine, Vol. 62, No. 6, 1998, pp. 783-792. doi: $10.1180 / 002646198548160$

[6] A. Mock and D. A. Jerram, "Crystal Size Distributions (CSD) in Three Dimensions: Insights from the 3D Reconstruction of a Highly Porphyritic Rhyolite," Journal of Petrology, Vol. 46, No. 8, 2005, pp. 1525-1541. doi:10.1093/petrology/egi024

[7] L. Qian, H. Toda, K. Uesugi, M. Kobayashi and T. Kobayashi, "Direct Observation and Image-Based Simula-n tion of Three-Dimensional Tortuous Crack Evolution inside Opaque Materials," Physical Review Letters, Vol. 100, No. 11, 2008, p. 115505. doi:10.1103/PhysRevLett.100.115505

[8] T. Q. Xiao, R. C. Chen, H. L. Xie, L. Rigon, R. Longo and E. Castelli, "Phase Retrieval in Quantitative X-Ray Microtomography with a Single Sample-to-Detector Distance," Optics Letters, Vol. 36, No. 9, 2011, pp. 1719 1721. doi:10.1364/OL.36.001719

[9] T. Q. Xiao, S. M. Shi, R. C. Chen, Y. L. Xue, Y. Q. Ren, G. H. Du, B. Deng and H. L. Xie, "X-Ray Microscopic Imaging of Low Z Material Wrapped by Strongly Absorbing Medium," Acta Physica Sinica, Vol. 57, No. 10, 2008, pp. 6319-6328.

[10] R. C. Chen, R. Longo, L. Rigon, F. Zanconati, A. De Pellegrin, F. Arfelli, D. Dreossi, R. H. Menk, E. Vallazza, T. Q. Xiao and E. Castelli, "Measurement of the Linear Attenuation Coefficients of Breast Tissues by Synchrotron Radiation Computed Tomography," Physics in Medicine and Biology, Vol. 55, No. 17, 2010, pp. 4993-5005. doi:10.1088/0031-9155/55/17/008 
[11] S. Mayo, A. Stevenson, S. Wilkins, D. Gao, S. Mookhoek, S. Meure, T. Hughes and J. Mardel, "X-Ray Phase-Contrast Tomography for Quantitative Characterisation of Self-Healing Polymers," The 7th Pacific Rim International Conference on Advanced Materials and Processing, Vol. 654-656, 2010, pp. 2322-2325.

[12] S. Yang, S. Furman and A. Tulloh, "A Data-Constrained 3D Model for Material Compositional Microstructures," Advanced Materials Research, Vol. 32, 2008, pp. 267270. doi:10.4028/www.scientific.net/AMR.32.267

[13] Y. S. Yang, A. Tulloh, I. Cole, S. Furman and A. Hughes, "A Data-Constrained Computational Model for Morphology Structures," Journal of the Australian Ceramics Society, Vol. 43, No. 2, 2007, pp. 159-164.

[14] H. J. Vinegar and S. L. Wellington, "Tomographic Imaging of 3-Phase Flow Experiments," Review of Scientific Instruments, Vol. 58, No. 1, 1987, pp. 96-107. doi:10.1063/1.1139522

[15] S. Yang, D. C. Gao, T. Muster, A. Tulloh, S. Furman, S. Mayo and A. Trinchi, "Microstructure of a Paint PrimerA Data-Constrained Modeling Analysis," The 7th Pacific Rim International Conference on Advanced Materials and Processing, Vol. 654-656, 2010, pp. 1686-1689.

[16] S. C. Mayo, A. M. Tulloh, A. Trinchi and Y. S. Yang, "Data-Constrained Microstructure Characterisation with Multi-Spectrum X-Ray," Microscopy and Microanalysis, Vol. 18, No. 3, 2012, pp. 524-530. doi:10.1017/S1431927612000323

[17] Y. Wang, Y. Yang, I. Cole, A. Trinchi and T. Xiao, "Investigation of the Microstructure of an Aqueously Corroded Zinc Wire by Data-Constrained Modelling with Multi-Energy X-Ray CT," Materials and Corrosion, Vol. 64, No. 3, 2013, pp. 180-184.

[18] A. Trinchi, Y. Yang, J. Huang, P. Falcaro, D. Buso and L. Cao, "Study of 3D Composition in a Nanoscale Sample Using Data-Constrained Modelling and Multi-Energy X-Ray CT," Modelling and Simulation in Materials Science and Engineering, Vol. 20, No. 1, 2012, p. 015013. doi:10.1088/0965-0393/20/1/015013

[19] Y. S. Yang, T. E. Gureyev, A. Tulloh, M. B. Clennell and M. Pervukhina, "Feasibility of a Data-Constrained Pre- diction of Hydrocarbon Reservoir Sandstone Microstructures," Measurement Science \& Technology, Vol. 21, No. 4, 2010, Article No. 047001.

[20] S. Yang and J. Taylor, "Model and Data Work Together to Reveal Microscopic Structures of Materials," SPIE Newsroom, 2010.

[21] W. H. Press, S. A. Teukolsky, W. T. Vetterling and B. P. Flannery, "Numerical Recipes: The Art of Scientific Computing,"3rd Edition, Cambridge University Press, 2007.

[22] T. Xiao, A. Bergamaschi, D. Dreossi, R. Longo, A. Olivo, S. Pani, L. Rigon, T. Rokvic, C. Venanzi and E. Castelli, "Effect of Spatial Coherence on Application of In-Line Phase Contrast Imaging to Synchrotron Radiation Mammography," Nuclear Instruments and Methods in Physics Research Section A: Accelerators, Spectrometers, Detectors and Associated Equipment, Vol. 548, No. 1, 2005, pp. 155-162. doi:10.1016/j.nima.2005.03.083

[23] G. Y. Zhu, S. C. Zhang and Y. B. Liang, "Palaeo Environment and the Distribution of H2S in the Triassic Feixianguan (T1f) Formation, Northeastern Sichuan Basin, South China," Journal of Petroleum Exploration and Development, Vol. 32, No. 2, 2005, pp. 65-69.

[24] J. Zhang and Y. G. Wang, "Characteristics of the Carbonate Evaporation Platform Margin Deposition of the Feixianguan Formation in the Hekou Region, Sichuan Basin," Journal of Natural Gas Industry, Vol. 23, No. 2, 2003, pp. 19-22.

[25] T. E. Gureyev, Y. Nesterets, D. Ternovski, D. Thompson, S. W. Wilkins, A. W. Stevenson, A. Sakellariou and J. A. Taylor, "Toolbox for Advanced X-Ray Image Processing," Vol. 8141, 2011, p. 81410B.

[26] D. Paganin, S. C. Mayo, T. E. Gureyev, P. R. Miller and S. W. Wilkins, "Simultaneous Phase and Amplitude Extraction from a Single Defocused Image of a Homogeneous Object," Journal of Microscopy-Oxford, Vol. 206, No. 1, 2002, pp. 33-40.

[27] Y. D. Wang, A. Stevenson, Y. S. Yang, A. Trinchi, S. Wilkins and T. Q. Xiao, "A Quantitative Study of Monochromatic X-Ray Transmission through Zinc Wires," Journal of Synchrotron Radiation, Vol. 19, 2012, pp. 827830. 